\title{
Psychotrope Substanzen
}

Psychotrope Substanzen dienen Menschen - wie auch manchen Tieren - dazu, positive Effekte in Bereichen wie Stimmung, Antrieb, Selbstwertgefühl, Leistungsvermögen oder Kontaktfähigkeit zu erzielen. Eng damit verbunden sind Gefahren des Missbrauches, der süchtigen Fehlentwicklung und der schweren Abhängigkeitserkrankung mit vielerlei desaströsen Auswirkungen. Hierzu gehören nicht nur die körperlichen Gesundheitsschäden, sondern auch die schweren psychosozialen Folgeerscheinungen wie Persönlichkeitsdepravation, intellektueller Abbau oder Zerstörung der beruflichen und sozialen Existenzgrundlagen. Viele der Einflüsse psychotroper Substanzen begünstigen kriminelle Verhaltensweisen und werden so Gegenstand psychiatrischer, psychopathologischer und kriminologischer Expertise. Hauptthemen dabei sind epidemiologische und kriminalstatistische Fragen, Beurteilung von Schuldfähigkeit und Prognose, die Behandlung und die Rückfallprävention. Deshalb stehen Probleme des schädlichen Gebrauches und der Suchterkrankungen im Umfeld von Alkohol, Rauschdrogen und sonstigen psychotropen Substanzen im Mittelpunkt dieses Themenheftes von FPPK.

Eine Einführung in die rechtlichen Aspekte gibt der Beitrag von W. Pfister, der aus Sicht eines Richters am BGH grundsätzlichen Fragestellungen nachgeht und insbesondere auf die nötige Zusammenarbeit zwischen psychowissenschaftlichen Sachverständigen und den verschiedenen Instanzen der Justiz hinweist, dies ganz im Sinne der Grundkonzeption unserer Zeitschrift. In den zentralen forensisch-psychiatrischen Bereich führt der Beitrag von E. Gouzoulis-Mayfranck über psychopathologische und kognitive Veränderungen unter Rauschdrogen. Dabei wird vor allem auf die subtile Analyse der kognitiven und affektiven Funktionsstörungen Wert gelegt, die der Autorin durch die experimentelle Psychoseforschung unter Einsatz von Stimulanzien und Psychotomimetika gut bekannt sind. Nicht die pauschale Be- rufung auf Drogeneinfluss und auf Berauschtheit, sondern nur die genaue psychopathologische Beschreibung der Tatzeitverfassung und nachweisbarer Leistungsausfälle erlauben Aussagen über das Einsichts- und Steuerungsvermögen.

Die epidemiologische Situation des Drogenkonsums in Deutschland und die damit verbundenen kriminologischen Auswirkungen werden im Artikel von Heilmann und Scherbaum analysiert. Die Veränderungen der letzten Jahre zeigen charakteristische Entwicklungen, dies auch im innerdeutschen Ost-West-Vergleich und im Prozess der europäischen Einigung, die mit einiger Latenz ihren Niederschlag in der Kriminalstatistik finden werden. Loddo et al. aus der Kölner Rechtsmedizin behandeln mit den sogenannten Ko-Tropfen eine kriminologisch höchst interessante Spezialform des Missbrauchs psychotroper Substanzen, die hier nicht dem Eigengebrauch dienen, sondern der Ermöglichung von Straftaten an anderen Personen.

Dem wichtigen Thema der Maßregelvollzugsbehandlung gemäß $\$ 64$ StGB widmet sich Schalast mit einem Beitrag über die Möglichkeiten suchtspezifischer medikamentöser Interventionen. Diese auch in der allgemeinen Psychiatrie und in der Suchtmedizin noch keineswegs durchgängig anerkannten Behandlungsformen bedürfen auch im forensischen Setting der Erprobung und Evaluation. Mit zunehmendem Wissen über äthopathogenetische, psychopharmakologische und pharmakogenetische Zusammenhänge bei der Entstehung, der Aufrechterhaltung und der therapeutischen Beeinflussung von Suchtverhalten steht zu hoffen, dass sich aus der Gesamtheit der betroffenen Patienten Subpopulationen definieren lassen, die von bestimmten Substanzen bzw. Kombinationen medikamentöser und verhaltenstherapeutischer Maßnahmen profitieren können.

Die therapeutischen Möglichkeiten im Rahmen der $\$ \$ 35,36$ des Betäubungsmittelgesetzes sind anfangs z. T. euphorisch überschätzt worden, auch hat sich im 
Laufe der Zeit ein erhebliches Missbrauchspotential gezeigt. Eine nüchterne Bewertung von Chancen und Gefahren nimmt der Beitrag von Gerasch vor, der sich auf einen besonders reichhaltigen staatsanwaltlichen Erfahrungshintergrund in Berlin stützen kann.

Auch in kommenden Themenheften von FPPK werden wir auf die Probleme durch psychotrope Substanzen im forensischen Umfeld zurückkommen. Wer sich aktuell weiter informieren möchte, sei auf den Themenschwerpunkt Suchterkrankungen im Septemberheft von „Der Nervenarzt“ hingewiesen [1-5], ferner auf die einschlägigen Beiträge im Berichtsband über die Jahrestagung der Gesellschaft für Kriminologie [6].

Henning Saß, Aachen

\section{Literatur}

1. Fallgatter AJ, Jakob CP (2009) Komorbidität von Suchterkrankungen und Aufmerksamkeitsdefizit-/Hyperaktivitätsstörung. Nervenarzt 80:1015-1021

2. Batra A, Friedrich HM, Lutz U (2009) Therapie der Nikotinabhängigkeit. Nervenarzt 80:1022-1029
3. Wölfling $\mathrm{K}$, Bühler $\mathrm{M}$, Leménager $\mathrm{T}$, Mörsen C, Mann K (2009) Glücksspiel und Internetsucht. Nervenarzt 80:10301039

4. Kiefer F, Großhans M (2009) Beitrag der Suchtforschung zum Verständnis der Adipositas. Nervenarzt 80:1040-1049
5. Kienast T, Roediger E, Kensche M (2009) Evidenzbasierte Psychotherapie. Suchtund Persönlichkeitsstörungen als Komorbidität, Nervenarzt 80:1050-1059

6. Haller R, Jehle JM (2009) Drogen-SuchtKriminalität. Verlag Godesberg $\mathrm{GmbH}$, Mönchengladbach 